\title{
A Formulation of Quantum Particle Swarm Optimization
}

\author{
Ichio Kikuchi and Akihito Kikuchi*
}

November 12, 2018

\begin{abstract}
In this article, we present our version of quantum particle swarm optimization. The objective function is the expectation value of the quantum mechanical Hamiltonian, as the sum of the kinetic term $\left(-h^{2} \frac{d^{2}}{d x^{2}}\right)$ and the polynomial objective function $(V(x))$. The quantum mechanical feature in the system is represented by the set of expectation values $\left(\phi\left|x^{i} u^{j}\right| \phi\right)$ for the possible combination of the kinetic momentum operator $\left(u=h \frac{d}{d x}\right)$ and the positional operator $(x)$. These elements should satisfy the semi-definite condition of a matrix which is the representation of the uncertainty principle. The Plank constant $h$ is a control parameter which would switch from the quantum mechanical case to the classical case, and vice versa. In order to get the optimum, these quantum mechanical expectation values are assumed to be the particle coordinates and propagated by the particle swarm optimization with the restriction. We present a simple example of the computational process.
\end{abstract}

\section{Formalism}

In this article, we present an extension of particle swarm optimization toward quantum mechanics.

In preceding work, we have built a kind of quantum computation[1]. This is an extension of "polynomial optimization", which is now applied in the optimization (not of the quantum mechanical context) and based on the moment problem[2]. (N.B. The works of polynomial optimization use the term "moment" as the integral of the form $\int x^{n} d \mu$ by means of some measure $\mu$, but not as the quantum mechanical kinetic momentum. ) Although the formalism presented in our previous study was solved by symbolic computation, the algorithm for the numerical solution shall lead to the quantized version of the particle optimization in the classical sense[3].

Let us review the algorithm of quantum computation in [1] through a simple model of the harmonic oscillator. The Hamiltonian is given by

$$
H=p^{2}+x^{2},
$$

*A.K. is the corresponding author: akihito_kikuchi@gakushikai.jp 
where $p$ and $x$ represent the kinetic momentum and the position. The commutator between $p$ and $x$ is given by

$$
[p, x]=-\sqrt{-1} h .
$$

It causes the computation in the complex number. So we would rather make use of $u=h \frac{d}{d x}$, instead of $p$, in order to do in the real numbers.

Now we represent the expectation values of the operator in the following way:

$$
M[x]=(\phi|x| \phi) .
$$

$\phi$ is the wave function, but we do not solve for it in our algorithm.

These relations are valid:

$$
\begin{gathered}
M[-A]=-M[A], \\
M[A \cdot B] \neq M[A] \cdot M[B], \\
M\left[x^{2}\right] \geq M[x] \cdot M[x], \\
M\left[-u^{2}\right] \geq M[u] \cdot M[u],
\end{gathered}
$$

and

$$
[u, x]=h .
$$

The first of them is the linearity with respect to the constant number. The relations from the second to the fourth are the feature of quantum mechanics. The fifth is the commutator, where the Plank constant $h$ could be an arbitrary scaling parameter.

Let us inspect the following quadratic form:

$$
\int[(a u+b x+c) \phi][(a u+b x+c) \phi] d x(\geq 0) .
$$

This quadratic form is rewritten as

$$
\begin{aligned}
& -a^{2} M[u u]+b^{2} M[x x]+c^{2} M[1]+a \cdot b(-M[u x]+M[x u])(10 \\
& +0 \cdot a \cdot c M[u]+2 \cdot b \cdot c M[x] \\
= & -a^{2} M[u u]+b^{2} M[x x]+c^{2} M[1]-h \cdot a \cdot b \\
& +0 \cdot a \cdot c M[u]+2 \cdot b \cdot c M[x] \\
= & \left(\begin{array}{lll}
c & b & a
\end{array}\right)\left(\begin{array}{lll}
M[1] & M[x] & 0 \cdot M[u] \\
M[x] & M[x x] & -h / 2 \\
0 \cdot M[u] & -h / 2 & M[-u u]
\end{array}\right)\left(\begin{array}{l}
c \\
b \\
a
\end{array}\right)(\geq 0)
\end{aligned}
$$

Here we use the relations:

$$
\begin{gathered}
M[1]=\int \phi \cdot \phi d x=1 . \\
M[u]=\int \phi\left(h \frac{d}{d x} \phi\right) d x=\frac{1}{2} \int\left(h \frac{d}{d x} \phi^{2}\right) d x=0 . \\
M[u u]=\int \phi\left(h^{2} \frac{d^{2}}{d x^{2}} \phi\right) d x . \\
M[x u]=\int \phi\left(x \cdot h \frac{d}{d x} \phi\right) d x
\end{gathered}
$$




$$
M[u x]=\int \phi \cdot\left(h \frac{d}{d x}(x \phi)\right) d x
$$

and

$$
M[x u]=M[u x]-h .
$$

The semi-positive definiteness of the quadratic form is replaced by that of the matrix in the last line in (11). This matrix is the so-called moment matrix, and it is now given as:

$$
M=\left(\begin{array}{lll}
1 & M[x] & 0 \\
M[x] & M[x x] & -h / 2 \\
0 & -h / 2 & M[-u u]
\end{array}\right)
$$

Semi-positive definiteness of the truncated moment matrix demands us these relations:

$$
\begin{gathered}
M[x x] \geq 0, \\
M[-u u] \geq 0, \\
M[x x] M[-u u]-h^{2} / 4 \geq 0, \\
M[x x]-M[x] M[x] \geq 0,
\end{gathered}
$$

and

$$
\operatorname{det}(M) \geq 0 \text {. }
$$

They are not the semi-positive definiteness of the moment matrix itself, but the semi-positiveness of the determinants of the minors taken on the diagonal. With this condition, the optimum of $E=M[-u u]+M[x x]$ is obtained at $M[x x]=M[-u u]=h / 2$.

We should note that the matrix $M$ is infinite dimensional and that the current representation is an approximation for simplicity. In order to fully describe the feature of the wave-function, we need the infinite order of the moment matrix: the commutators generate infinite numbers of terms.

Now we have represented the problem as a polynomial optimization, where the objective function and the restraints are given by polynomials. The numerical solution can be executed by the particle swarm optimization (PSO). It is a kind of molecular dynamics: the particles move in a way analogical to the behavior of a group of flying birds; each member of the group (indexed by $j$ ) seeks the optimum point, varying the velocity $v_{j}$ and the position $s^{j}$, being attracted by the past best position of itself $p_{\text {best }}^{j}$ and by that of the past group best $g_{\text {best }}^{j}$. The time-evolution of the particle system is executed in the discrete time steps; the velocity $v^{j}$ of the particle is curbed by the friction and every member of the group will cease to move and assemble at the unique best position. The evolution is given by

$$
v^{j} \leftarrow w \cdot v^{j}+C_{1} \cdot r_{1} \cdot\left(p_{\text {best }}^{j}-s^{j}\right)+C_{2} \cdot r_{1} \cdot\left(g_{\text {best }}^{j}-s^{j}\right),
$$

and

$$
s^{j} \leftarrow s^{j}+\cdot v^{j}
$$

The constant parameter $w$ is the inertia which leads the group to a halt in the end; $C_{1}$ and $C_{2}$ are the constant parameters which determine the extent of the jump of the velocities and the positions from one step to the 
next; the alternation of the velocity and the positions are also regulated by random numbers $r_{1}$ and $r_{2}$ of the uniform distribution in [0,1]. The individual best and the group best should be updated whenever they are detected.

In our quantum swarm optimization, the coordinates of the particle are allotted to the expectation of the quantum mechanical operators $(M[x]$ $M[x x]$ and $M[u u])$. Although the classical velocity of a particle might be related to the quantum expectation values of the momentum, we propagate the quantum mechanical expectation values independently, giving the virtual velocity to each of them. (In fact, the expectation value of the kinetic momentum in the optimum of the current problem is zero and we neglect it because the problem is to find the bound state at the bottom of the parabolic potential.)

We should impose the constraints concerning the polynomial inequalities which are given in (18)-(22). For this purpose, we add the penalty functions to the objective function in the following form.

$$
\begin{aligned}
\Theta(x ; p(x)) & =0 \text { when } p(x) \geq 0, \\
& =1 \text { when } p(x)<0 .
\end{aligned}
$$

The result of the numerical experiment (by means of the standard style of PSO) for the harmonic oscillator is given in Table 1. The Plank constant is taken to be $h=1$. The table shows the group best of the expectation values of $M[x] M[x x]$ and $M[u u]$. The parameters in PSO are chosen to be $w=0.7$ and $C_{1}=C_{2}=1.4$, and 30 sampling particles are used. After the $250-300$ times of the iteration, the swarm has arrived at the optimum. 


\begin{tabular}{|c|c|c|c|c|c|c|c|}
\hline & $\mathrm{M}[\mathrm{x}]$ & & & $\begin{array}{l}-\mathrm{M}[\mathrm{uu}] \\
+\mathrm{M}[\mathrm{xx}]\end{array}$ & & & \\
\hline 0 & -0.5227 & -0.6507 & .6890 & 1.3398 & & & \\
\hline 20 & & -0.5 & & & & & \\
\hline 40 & 242 & -0.5 & & 008 & & & \\
\hline 60 & 037 & -0.5 & & & & & \\
\hline 80 & & & & & & & \\
\hline .00 & 05 & -0.5 & & & & & \\
\hline & & & & & & & \\
\hline \pm 0 & 01 & -0.5 & & & 2. & & -11 \\
\hline 50 & & & & & & & \\
\hline & & -0.5 & & & 1.7 & & \\
\hline 0 & 01 & -0.50 & 97 & 000 & 1.1 & & -09 \\
\hline & 02 & -0.5 & & & 3.7 & & -09 \\
\hline \pm 0 & -0.0001 & -0.5000 & 0.5 & .0000 & 7.3 & -11 & -11 \\
\hline & & -0.5 & & & 7.0 & & -11 \\
\hline & & -0.5 & & & 2.0 & -12 & -12 \\
\hline & & & & & 1.0 & & -12 \\
\hline & & -0.5 & & & $1.0774 \mathrm{e}-12$ & $3 e-12$ & $1.7442 \mathrm{e}-12$ \\
\hline & 0.0000 & -0.5000 & 0.5000 & 1.0000 & $2.3645 e-13$ & $2.9798 e-13$ & $2.9793 e-13$ \\
\hline
\end{tabular}

Table 1: The evolution of the quantum mechanical expectation values in the particle swarm optimization in the example problem. The model is the harmonic oscillator and the Plank constant is taken to be $h=1$. Each row shows the results of every twenty iterations, in which the group best values and the valiance taken from the swarm are denoted by $\mathrm{M}[\mathrm{]}$ and $\operatorname{VAR}($ ).

The current example is a simple model. But we can extend it in the multi-dimensional case and we can apply it to the more complicated polynomial objective function. In this case,, we should construct the matrix (which depicts the uncertaintynty) from and the commutator in equation (8) and the quadratic form

$$
\int\left[\sum_{p, q} x^{p} u^{q} \phi\right]\left[\sum_{p, q} x^{p} u^{q} \phi\right] d x(\geq 0) .
$$

\section{Summary}

In this article, we present our version of quantum particle swarm optimization. The objective function is the expectation value $(\phi|H| \phi)$ of the quantum mechanical Hamiltonian $H=K+V$, represented by the kinetic term $\left(K=-h^{2} \frac{d^{2}}{d x^{2}}\right.$ ) and the polynomial objective function $(V(x))$. The quantum mechanical feature in the system is represented by the set of expectation values for the possible combination of the momentum $\left(u=h \frac{d}{d x}\right)$ and the positional operator $(x)$, as is given by $\left(\phi\left|x^{i} u^{j}\right| \phi\right)$. The wave-function $\phi$ is not directly determined, but the profile is given by these expectation values. These expectation values should satisfy the semi-definite condi- 
tion of a matrix, which is the representation of the uncertainty principle. The optimum of the energy (the kinetic term and the object function) should be sought with the restriction of the semi-positive definiteness of that matrix. For the matrix is in principle infinite dimensional, and we should approximate it by a finite dimensional one. With this approximation, the given problem is given by the set of polynomial inequalities. We can solve it by the standard process of particle swarm optimization and we can add more restrictions to the basic set of the inequalities. By using a larger matrix, we can improve the result. We use the Plank constant (in the commutator between the momentum and the positional operator) as the parameter which regulates the quantum mechanical uncertainty in the computation. In the limit of $h=0$, the expectation value of the kinetic term $(\phi|u u| \phi)$ is zero. So we could expect the almost classical result for the optimization of the potential function $V$ when we use the small $h$ in the numerical computation.

\section{References}

[1] I. Kikuchi and A. Kikuchi, "Polynomial Optimization and Quantifier Elimination in Quantum Mechanics", OSF Preprints, https://doi.org/10.31219/osf.io/enhzt, 2018.

[2] M. Mevissen, "Introduction to concepts and advances in polynomial optimization", Institute for Operations Research, ETH, Zurich, 2007.

[3] Federico Marini, Beata Walczak, "Particle swarm optimization (PSO). A tutorial", Chemometrics and Intelligent Laboratory Systems 149(2015) 153-165. 